\title{
Tunable Giant Anomalous Hall Angle in Perpendicular Multilayers by Interfacial Orbital Hybridization
}

Jingyan Zhang, ${ }^{1}$ Wenlin Peng, ${ }^{1}$ Guanghua $\mathrm{Yu},{ }^{1 *}$ Zhanbing He, ${ }^{2}$ Feng Yang, ${ }^{3}$ Wei Ji, ${ }^{3}$ Chen $\mathrm{Hu},{ }^{4}$ and Shouguo Wang ${ }^{1,2 *}$

${ }^{1}$ Beijing Advanced Innovation Center for Materials Genome Engineering, Department of Materials Physics \& Chemistry, University of Science and Technology Beijing, Beijing 100083, China

${ }^{2}$ State Key Laboratory for Advanced Metals and Materials, University of Science and Technology Beijing, Beijing 100083, China

${ }^{3}$ Department of Physics, Renmin University of China, Beijing 100872, China

${ }^{4}$ Department of Physics, McGill University, Montreal, H3A2T8, Canada

*Corresponding author E-mail: ghyu@mater.ustb.edu.cn and sgwang@ustb.edu.cn

\section{Table of Contents}

S1. Magnetic properties for the multilayered structure with various period numbers

S2. Microstructural characterization for the multilayers

S3. Hall measurements for the Co/Pt multilayers with different metal/oxide interfaces

S4. First-principles calculation and the analysis of scattering mechanisms 
S1: Magnetic properties for the multilayered structure

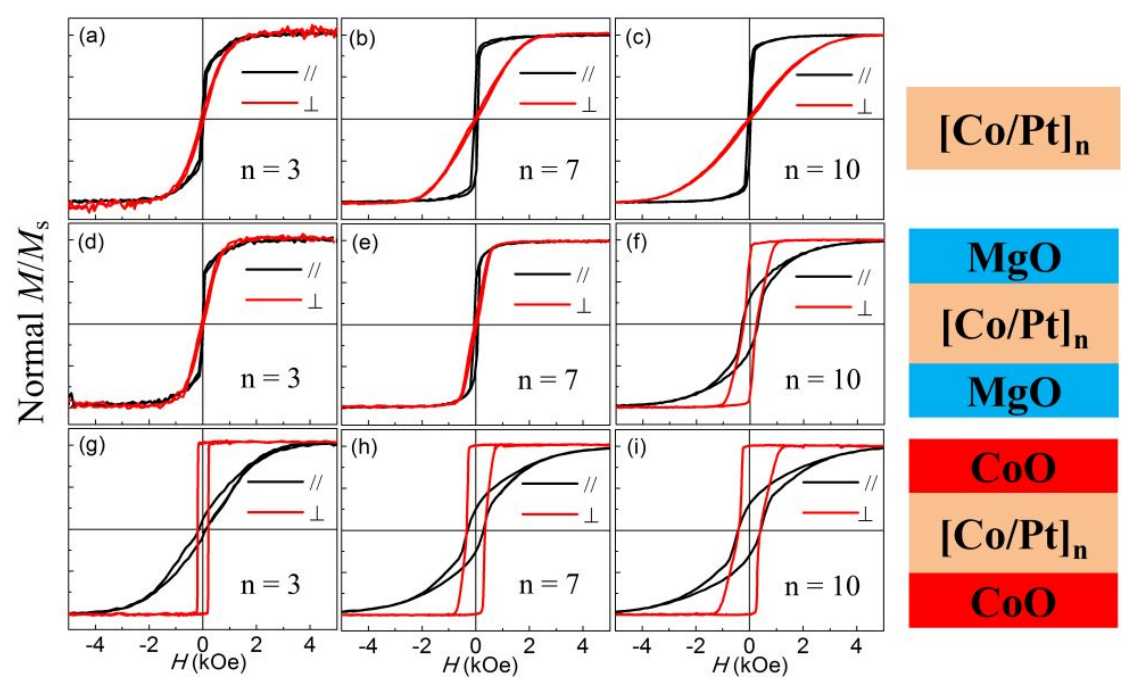

Fig. S1 (a) - (i) Perpendicular (red line) and in-plane (black line) $M / M_{\mathrm{s}}-\mathrm{H}$ loops for the multilayered structure $[\mathrm{Co} / \mathrm{Pt}]_{\mathrm{n}}, \mathrm{MgO} /[\mathrm{Co} / \mathrm{Pt}]_{\mathrm{n}} / \mathrm{MgO}$ and $\mathrm{CoO} /[\mathrm{Co} / \mathrm{Pt}]_{\mathrm{n}} / \mathrm{CoO}$ with $\mathrm{n}=3,7,10$.

In our work, the magnetic hysteresis loops for the samples with structures of $[\mathrm{Co} / \mathrm{Pt}]_{\mathrm{n}}, \mathrm{MgO} /[\mathrm{Co} / \mathrm{Pt}]_{\mathrm{n}} / \mathrm{MgO}$ and $\mathrm{CoO} /[\mathrm{Co} / \mathrm{Pt}]_{\mathrm{n}} / \mathrm{CoO}$ were measured. The samples with $\mathrm{n}=3,7,10$ were selected. Fig. S1 (a) - (i) shows the perpendicular (red line) and in-plane (black line) $M / M_{\mathrm{s}}-\mathrm{H}$ loops for the typical samples, respectively. For $[\mathrm{Co} / \mathrm{Pt}]_{\mathrm{n}}$ multilayers, the easy magnetic axis always keeps in plane with $n=3-10$. The magnetic anisotropy $\left(K_{\text {eff }}\right)$ decreases significantly with increasing n. For $\mathrm{MgO} /[\mathrm{Co} / \mathrm{Pt}]_{\mathrm{n}} / \mathrm{MgO}$ multilayers, the easy magnetic axis keeps in plane with $\mathrm{n}$ is below 7. When $\mathrm{n}$ is beyond $7(\mathrm{n}=8-10)$, the perpendicular magnetic anisotropy (PMA) was observed in the multilayers. Fig. S1 (g) - (i) shows the hysteresis loops for $\mathrm{CoO} /[\mathrm{Co} / \mathrm{Pt}]_{\mathrm{n}} / \mathrm{CoO}$ multilayers, respectively. It is worthy to point out that the samples with $\mathrm{CoO}$ layers exhibit PMA with increasing $\mathrm{n}$ from 3 to 10 . I $\mathrm{t}$ is clearly seen that the $\mathrm{M}-\mathrm{H}$ loops show obvious hysteresis for the samples with $\mathrm{CoO}$, indicating a weaker PMA with increasing $\mathrm{n}$.

\section{S2: Microstructural characterization for the multilayers}

The crystallographic structure of the multilayers was determined by conventional high-resolution transmission electron microscopy (phase-contrast, HRTEM). Fig. S2 
(a) presents high resolution cross-sectional TEM for the $\mathrm{CoO} /[\mathrm{Co} / \mathrm{Pt}]_{7} / \mathrm{CoO}$ multilayers. Further HRTEM observation shown in Fig. S2 (a) demonstrates the crystalline nature of the sandwiched structure. A smooth interface is qualitatively observed between $[\mathrm{Co} / \mathrm{Pt}]_{7}$ multilayers and $\mathrm{CoO}$ layer. Three regions, referred to as 1 , 2, and 3, are selected to further examine the metal/oxide interface as shown in Fig. S2 (b) $\sim($ d). The interplanar spacing of $0.24 \mathrm{~nm}$ and $0.22 \mathrm{~nm}$ corresponds to fcc $\mathrm{CoO}$ (111) and CoPt (111) plane, respectively. In Fig. S2 (b), the top CoO atomic layers adjacent $[\mathrm{Co} / \mathrm{Pt}]_{\mathrm{n}}$ multilayers exhibits crystalline structure, in good agreement with the previous study. A similar observation at bottom interface is presented in Fig. S2 (d). The results mentioned above indicate that a local epitaxial relationship of $\{111\} \mathrm{CoO} / /\{111\} \mathrm{CoPt} / /\{111\} \mathrm{CoO}$ or $\quad<110>\mathrm{CoO} / /<110>\mathrm{CoPt} / /<110>\mathrm{CoPt}$, showing highly textured metal/oxide interfaces. The Morie fringes in Fig. S2 (d) show a minor rotation of $\mathrm{CoO}$ (111) planes at an angle of $11^{\circ}$, which can partially be attributed to the oxide layer roughness.

Cs-corrected high-angle annular dark-field STEM (HAADF-STEM) was chosen to characterize the multilayered structure, focusing on the $\mathrm{Co} / \mathrm{Pt}$ interfaces. Fig. S3 presents the HAADF-STEM image for the $\mathrm{CoO} /[\mathrm{Co} / \mathrm{Pt}]_{7} / \mathrm{CoO}$ sandwiched structure. In this image, Co and Pt atomic layer are separated clearly due to large differences in atomic numbers $\left(\mathrm{Z}_{\mathrm{Co}}=27\right.$ and $\left.\mathrm{Z}_{\mathrm{Pt}}=78\right)$. When examining the interface between Co and Pt layer, dark and bright stripes can be alternately observed in Fig. S3, indicating continuous $\mathrm{Co} / \mathrm{Pt}(\mathrm{Pt} / \mathrm{Co})$ interface $(\mathrm{Co}$ and $\mathrm{Pt}$ layer thickness is only $0.6 \mathrm{~nm})$ in our mutlilayes by magnetron sputtering. More details about microstructural characterization can be accessed by the parallel acquisition of HAADF-STEM signals along the growth direction perpendicular atomic planes shown in inset of Fig. S3. HAADF image intensity peak show a alternative change between higher intensity for $\mathrm{Pt}$ and lower intensity for Co. The final contrast of the image intensity differentiates clearly between $\mathrm{Co}$ and $\mathrm{Pt}$ atomic layer though is not sensitive to oxygen in $\mathrm{CoO}$ due to its low atomic number compared with Co and Pt. The 'wave-like' diagram can be observed in inset of Fig. S3, where crest and valley corresponds to Pt and Co layers, respectively. The distance of the adjacent valley can be estimated as $1.13 \mathrm{~nm}$, which is approximately the nominal thickness of $\mathrm{Co} / \mathrm{Pt}$ hybrid bilayers (period number $\sim 1$ ). 
Moreover, the intensity of Pt layer adjacent oxide layer is lower than that of Pt layer inside the $[\mathrm{Co} / \mathrm{Pt}]_{\mathrm{n}}$ multilayers. It should be due to higher intensity from $\mathrm{Co}$ in $\mathrm{CoO}$ layer at interface and from the background. Therefore, a sharp and continuous $\mathrm{Co} / \mathrm{Pt}$ $(\mathrm{Pt} / \mathrm{Co})$ interfaces can be observed in our multilayered structure.

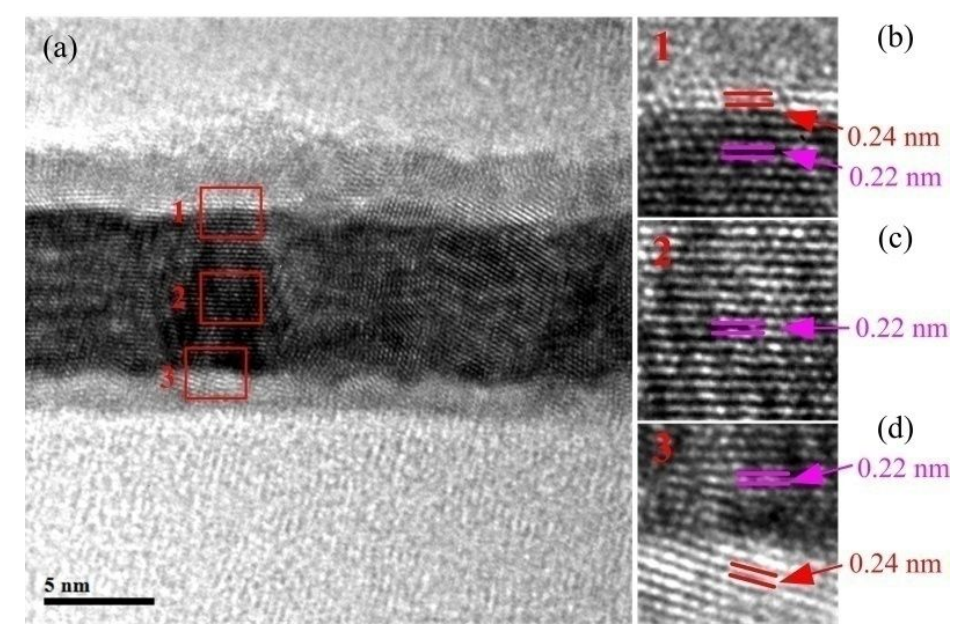

Fig. S2 (a) High resolution cross-sectional TEM image for the $\mathrm{CoO} /[\mathrm{Co} / \mathrm{Pt}]_{7} / \mathrm{CoO}$ multilayers. (b) , (c) and (d) HRTEM images from regions labeled 1, 2, and 3 in panel (a), respectively.

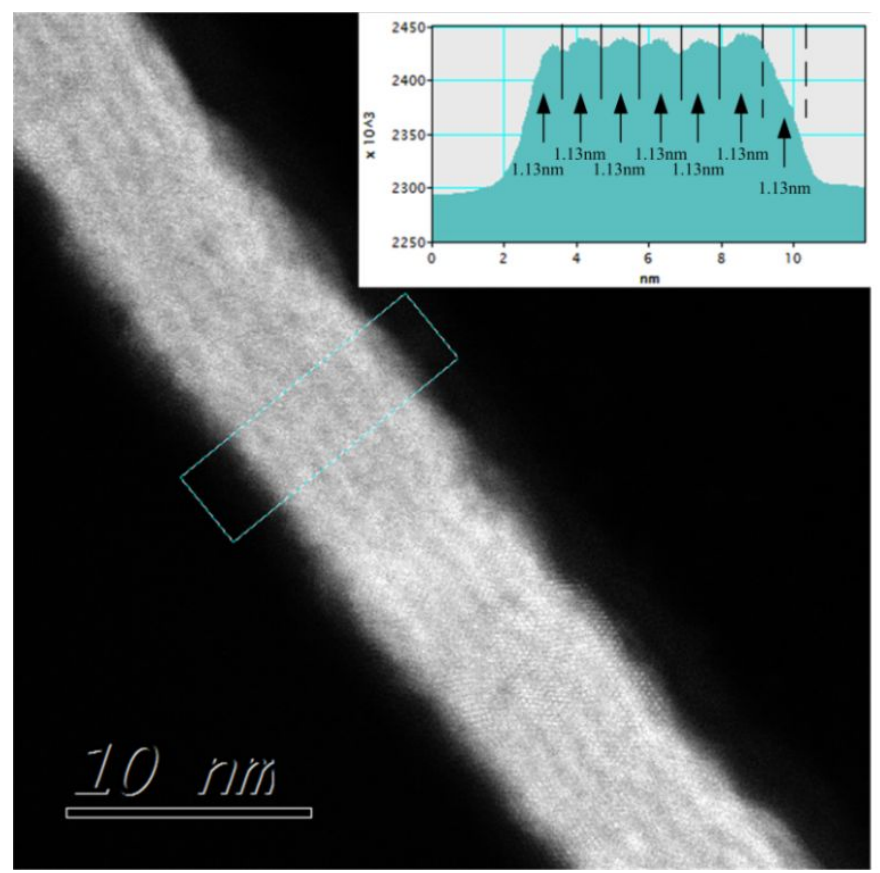

Fig. S3 HAADF-STEM for the $\mathrm{CoO} /[\mathrm{Co} / \mathrm{Pt}]_{7} / \mathrm{CoO}$ multilayers. Inset: intensity line scans perpendicular to $\mathrm{Co}$, and $\mathrm{Pt}$ atomic planes, marked schematically by the green rectangles in the HAADF-STEM image. 
S3: Hall measurements for the $\mathrm{Co} / \mathrm{Pt}$ multilayers with different metal/oxide interfaces

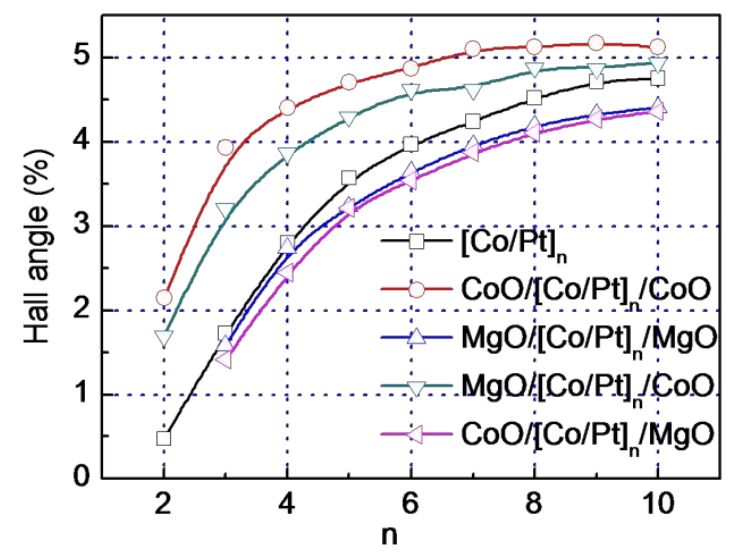

Fig. S4 Anomalous Hall angle $\theta$ as a function of the period number $\mathrm{n}$ for the multilayers with various metal/oxide interfaces.
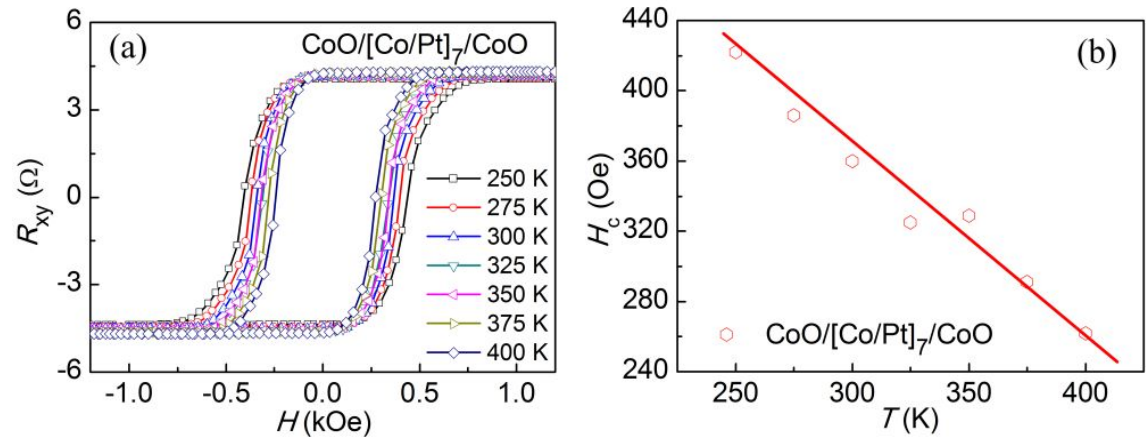

Fig. S5 (a) Hall loops for the $\mathrm{CoO} /[\mathrm{Co} / \mathrm{Pt}]_{7} / \mathrm{CoO}$ multilayers at a wide temperature region $(250 \mathrm{~K}$ $400 \mathrm{~K})$, (b) the coercivity $\left(H_{\mathrm{c}}\right)$ as a function of temperature for the sample $\mathrm{CoO} /[\mathrm{Co} / \mathrm{Pt}]_{7} / \mathrm{CoO}$.

In our work, the $[\mathrm{Co} / \mathrm{Pt}]_{\mathrm{n}}$ multilayers was sandwiched by oxide layers $(\mathrm{MgO}$ or $\mathrm{CoO})$. The $\theta$ value for the multilayers with $\mathrm{CoO}$ is larger than that of the multilayers with $\mathrm{MgO}$. To further investigate the effect of the bottom oxide/[Co/Pt $]_{\mathrm{n}}$ interface and top $[\mathrm{Co} / \mathrm{Pt}]_{\mathrm{n}} /$ oxide interface on $\theta$ value, the magnetic transport measurements for the $[\mathrm{Co} / \mathrm{Pt}]_{\mathrm{n}}$ multilayers sandwiched by various oxide layers were carried out. Fig. S4 presents the value of $\theta$ as a function of the period number (n) for the multilayers with structures of $[\mathrm{Co} / \mathrm{Pt}]_{\mathrm{n}}, \mathrm{CoO} /[\mathrm{Co} / \mathrm{Pt}]_{\mathrm{n}} / \mathrm{CoO}, \mathrm{MgO} /[\mathrm{Co} / \mathrm{Pt}]_{\mathrm{n}} / \mathrm{MgO}, \mathrm{MgO} /[\mathrm{Co} / \mathrm{Pt}]_{\mathrm{n}} / \mathrm{CoO}$, and $\mathrm{CoO} /[\mathrm{Co} / \mathrm{Pt}]_{\mathrm{n}} / \mathrm{MgO}$, respectively. For the samples with various oxide/metal interfaces, $\theta$ shows a significant increase. Compared with the multilayers with 
$[\mathrm{Co} / \mathrm{Pt}]_{\mathrm{n}} / \mathrm{MgO}$ interfaces (for example, $\mathrm{CoO} /[\mathrm{Co} / \mathrm{Pt}]_{\mathrm{n}} / \mathrm{MgO}$ ), the $\theta$ value for the $\mathrm{CoO} /[\mathrm{Co} / \mathrm{Pt}]_{\mathrm{n}} / \mathrm{CoO}$ multilayers is always larger. Moreover, $\theta$ for the $\mathrm{MgO} /[\mathrm{Co} / \mathrm{Pt}]_{\mathrm{n}} / \mathrm{CoO}$ multilayers is also larger than that of the $\mathrm{CoO} /[\mathrm{Co} / \mathrm{Pt}]_{\mathrm{n}} / \mathrm{MgO}$ multilayers. It suggests that $[\mathrm{Co} / \mathrm{Pt}]_{\mathrm{n}} / \mathrm{CoO}$ interface plays a more important role on Hall angle compared with $\mathrm{CoO} /[\mathrm{Co} / \mathrm{Pt}]_{\mathrm{n}}$ interface. It is reasonable to conclude that both $[\mathrm{Co} / \mathrm{Pt}]_{\mathrm{n}} / \mathrm{CoO}$ and $\mathrm{CoO} /[\mathrm{Co} / \mathrm{Pt}]_{\mathrm{n}}$ interfaces make larger $\mathrm{AHE}$ behavior. Those results should be related to the interfacial behavior confirmed by microstructural characterization shown in Fig. S2. Fig. S5 (a) presents the Hall loops for the multilayers $\mathrm{CoO} /[\mathrm{Co} / \mathrm{Pt}]_{7} / \mathrm{CoO}$ at various temperatures. It shows the easy magnetic axis of the multilayers always keep out of plane for various temperatures. Good PMA can be observed for the multilayers with wide temperature region $(250 \mathrm{~K} \sim 400 \mathrm{~K})$, where the remanent states in our Hall loop at zero fields will be used for binary information storage and the AHE resistance allows retrieval of the magnetic state of the device. Hall loops for the multilayers at various temperatures show a slight change of the coercivity $\left(H_{\mathrm{c}}\right)$. The temperature dependence of $H_{\mathrm{c}}$ for $\mathrm{CoO} /[\mathrm{Co} / \mathrm{Pt}]_{7} / \mathrm{CoO}$ multilayers was shown in Fig. S5 (b). $\mathrm{H}_{\mathrm{c}}$ decreases significantly with increasing temperature. It shows that $H_{\mathrm{c}}$ is always larger than $250 \mathrm{Oe}(250 \mathrm{Oe} \sim 420 \mathrm{Oe})$ during temperature region $(250 \mathrm{~K} \sim 400 \mathrm{~K})$, where the Hall resistance can be turned at the magnetization switching process. This result will make a wide work temperature region for the further storage device.

S4: First-principles calculation and the analysis of scattering mechanisms

Fig. S6 (a) presents the total energy $E_{\text {tot. }}$ for $[\mathrm{Co} / \mathrm{Pt}]_{1}$ multilayers with two different configurations, where two configurations are A (homocrystalline texture) and $\mathrm{B}$ (anti-homocrystalline texture) shown in inset of the figure. The $\mathrm{E}_{\text {tot. }}$ value for $\mathrm{A}$ is lower than that of configuration $\mathrm{B}$, indicating configuration $\mathrm{A}$ is more stable. To build more stable $\mathrm{Co} / \mathrm{Pt}$ multilayers structures, the $\mathrm{E}_{\text {tot. }}$ for the multilayers with the Co and Pt lattice constant was calculated shown in Table 1, respectively. With $\mathrm{n}$ increasing, $\mathrm{E}_{\text {tot. }}$ for the multilayers with Co lattice constant is always larger than that for the multilayers with Pt lattice constant. Therefore, homocrystalline texture with Pt lattice constant is stable in the first-principles calculation. The atomic configuration of 
$\mathrm{Co} / \mathrm{Pt}$ multilayers can be found in the left-bottom of Fig. S6. Fig. S6 (b) and (c) presents the interplanar spacing $(\mathrm{L})$ and atomic magnetic moment $(\mathrm{M})$ for each atomic layer with various period number $(n=1 \sim 4)$, respectively. A significant change of L value for two adjacent atomic layer can be observed, where $\mathrm{L}_{\mathrm{Pt}-\mathrm{Pt}}>\mathrm{L}_{\mathrm{Pt}-\mathrm{Co}}>\mathrm{L}_{\mathrm{Co}-\mathrm{Co}}$. The $\mathrm{M}$ value at Co atomic layer is larger than that at Pt atomic layer. The similar behavior about $\mathrm{L}$ and $\mathrm{M}$ can be also observed with increasing $\mathrm{n}$. The multilayers with $\mathrm{n}=2$ was selected as the basic configuration for the first-principles calculation, which is for the appropriate calculation.

Spin-orbit coupling (SOC) energy at metal/oxide interfaces with oxygen-poor state was discussed. Four interfacial configurations (A3: (Mg)O-Pt, A4: (Mg)O-Co, B3: (Co)O-Pt, B4: (Co)O-Co) are shown in Fig. S7 (a), where SOC energy at the oxygen-terminated interfaces (oxygen-rich) will be clarified here. Fig. S7 (b) and (c) presents the $\mathrm{E}_{\mathrm{soc}}$ value and $\Delta \mathrm{E}_{\mathrm{soc}}$ at each atomic layer for the mutlilayers with various metal/oxide interfaces (A3, A4, B3, and B4), respectively. For A3, $\mathrm{E}_{\mathrm{soc}}$ value at Pt-1 Pt-3 changes significantly compared with pure $[\mathrm{Co} / \mathrm{Pt}]_{2}$ multilayers. However, $\mathrm{E}_{\mathrm{soc}}$ value at Co-4 Co-6 has little change with introducing A4. The similar thing happens for both B3 (Co)O-Pt and B4 (Co)O-Co. To quantify the change of SOC energy for four interfacial configurations, $\Delta \mathrm{E}_{\mathrm{soc}}$ at each atomic layer was given. The value of $\Delta \mathrm{E}_{\mathrm{soc}}$ for $\mathrm{A} 3$ and $\mathrm{B} 3$ is approximately $0.02 \mathrm{eV}$ and $-0.03 \mathrm{eV}$, respectively. However, the $\Delta \mathrm{E}_{\mathrm{soc}}$ value for $\mathrm{A} 4$ and $\mathrm{B} 4$ is nearly zero $(-0.006 \mathrm{eV}$ and $-0.005 \mathrm{eV})$, respectively. Fig. $S 7$ (d) shows the $\Delta E_{\mathrm{soc}} / \mathrm{E}_{\mathrm{soc}}$ as a function of $\mathrm{n}$ for four multilayered configurations (A3, A4, B3, and B4), respectively. It shows that the $\Delta \mathrm{E}_{\mathrm{soc}} / \mathrm{E}_{\mathrm{soc}}$ value for four configurations decreases with $\mathrm{n}$ increasing. For example, $\Delta \mathrm{E}_{\mathrm{soc}} / \mathrm{E}_{\mathrm{soc}}$ for $\mathrm{A} 3$ is $0.33 \%$ with $\mathrm{n}=2$, and it changes to $0.068 \%$ with $\mathrm{n}=10$. The $\Delta \mathrm{E}_{\mathrm{soc}} / \mathrm{E}_{\mathrm{soc}}$ for $\mathrm{A} 4$ and $\mathrm{B} 4$ is far smaller. It indicates a weaker effect of interfacial structure on SOC energy when $n$ is high enough.

It is worthy to mention that metal (oxygen)-terminated interface has different role in interfacial SOC energy. For $[\mathrm{Co} / \mathrm{Pt}]_{\mathrm{n}}$ multilayers sandwiched by $\mathrm{MgO}$ layers, there are $\mathrm{Mg}$-terminated interface and O-terminated interface, including the following four configurations (A1: Mg-Co, A2: Mg-Pt, A3: (Mg)O-Pt, (Mg)O-Co). Fig. S7 (a) 
shows $\Delta \mathrm{E}_{\mathrm{soc}} / \mathrm{E}_{\mathrm{soc}}$ as a function of the period number (n) for $\mathrm{A} 1 \sim \mathrm{A} 4$, respectively. The value of $\Delta \mathrm{E}_{\mathrm{soc}} / \mathrm{E}_{\mathrm{soc}}$ for $\mathrm{A} 2$ decreases significantly with $\mathrm{n}$ increasing. However, the change of the $\Delta \mathrm{E}_{\mathrm{soc}} / \mathrm{E}_{\mathrm{soc}}$ value via $\mathrm{n}$ for $\mathrm{A} 1, \mathrm{~A} 3$ and $\mathrm{A} 4$ can be neglected compared with $\mathrm{A} 2$. For $\mathrm{CoO} /[\mathrm{Co} / \mathrm{Pt}]_{\mathrm{n}} / \mathrm{CoO}$ multilayers, the change of the $\Delta \mathrm{E}_{\mathrm{soc}} / \mathrm{E}_{\mathrm{soc}}$ value via $\mathrm{n}$ for B2 is larger than that for B1, B3 and B4 as shown in Fig. S7 (b).

(a)
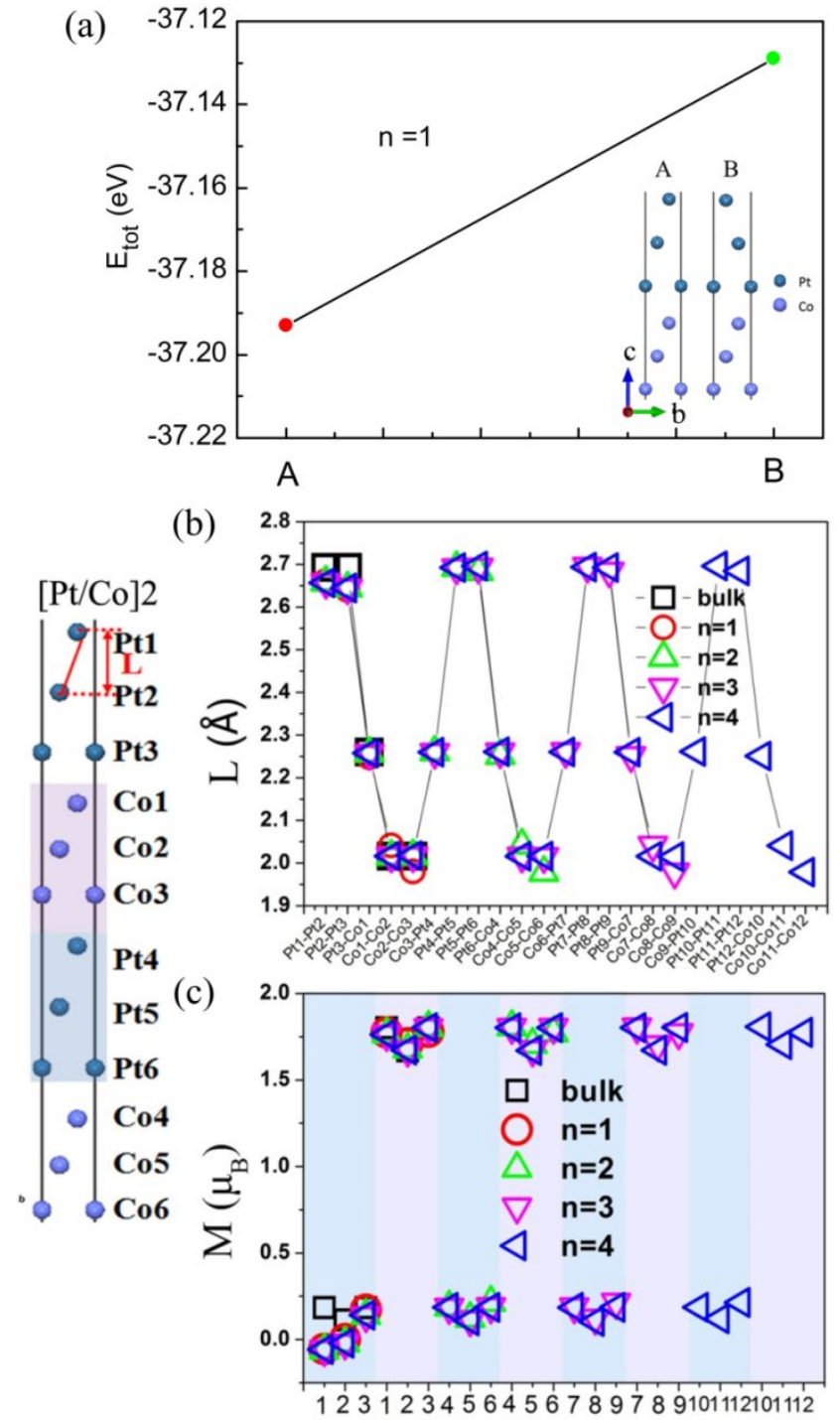

Fig. S6 (a) The total energy $\left(E_{\text {tot }}\right)$ of the Co/Pt multilayers with $n=1$ for two configuration shown in inset. Inset: two structural configurations A and B. (b) and (c) The interplanar spacing (L) and atomic magnetic moment (M) at each layer for the Co/Pt multilayers with different $\mathrm{n}(\mathrm{n}=1 \sim 4)$, respectively. 
Table 1

\begin{tabular}{cccc}
\hline & $n$ & $\mathrm{E}_{\text {tot }}($ configuration A, eV) & $\mathrm{E}_{\text {tot }}$ (configuration B, eV) \\
\hline & 1 & -36.268 & -36.267 \\
Co lattice constant & 2 & -73.612 & -73.609 \\
& 3 & -110.961 & -110.952 \\
& 1 & -37.193 & -37.129 \\
Pt lattice constant & 2 & -76.066 & -76.019 \\
& 3 & -114.941 & -114.908 \\
\hline
\end{tabular}

(a)

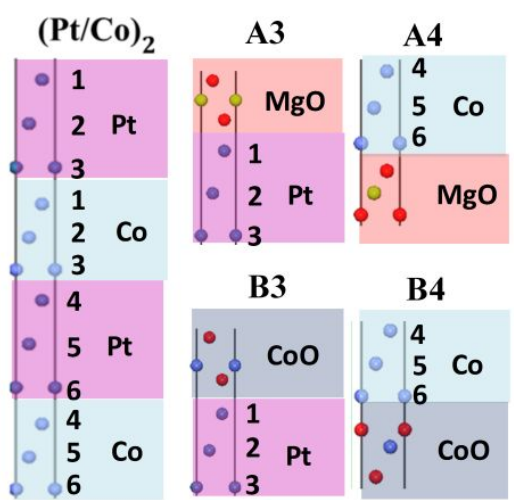

(c)

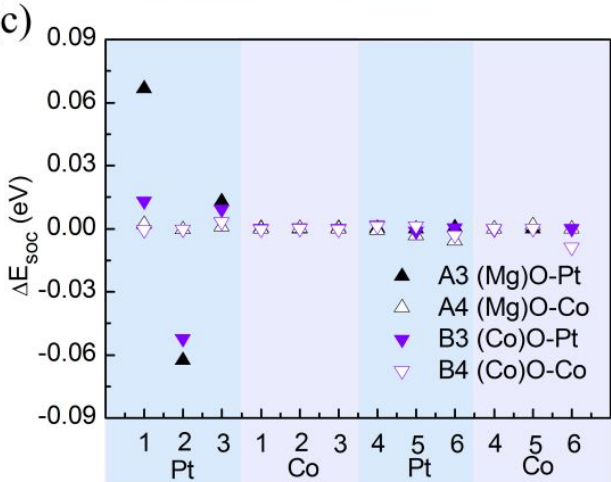

(b)

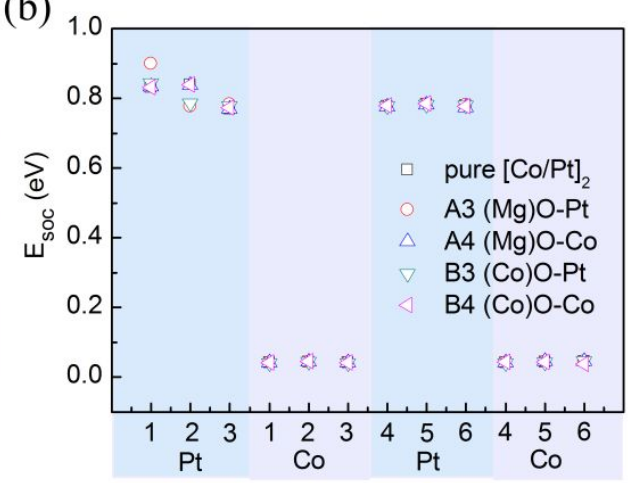

(d)

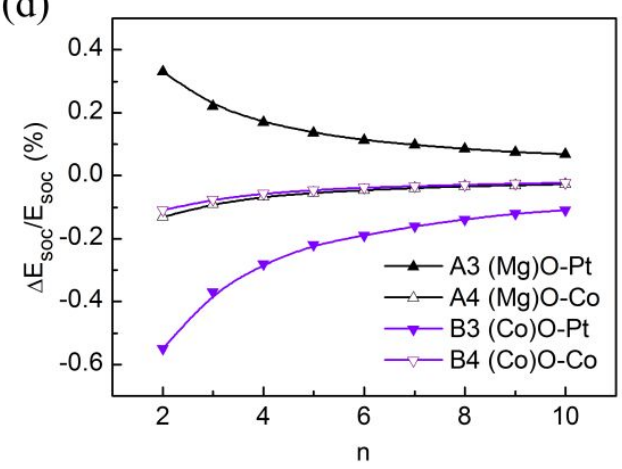

Fig. S7 (a) Four metal/oxide interface configurations (A3, A4, B3, and B4) for interfacial oxygen-rich states of the first-principles calculation. (b) Spin orbit coupling (SOC) energy for the atomic layer for four oxide/metal interfaces. (c) The variation of SOC energy at each atomic layer for four configurations. (d) The variation of SOC energy as a function of the period number for the four configurations with $\mathrm{CoO}$ or $\mathrm{MgO}$. 


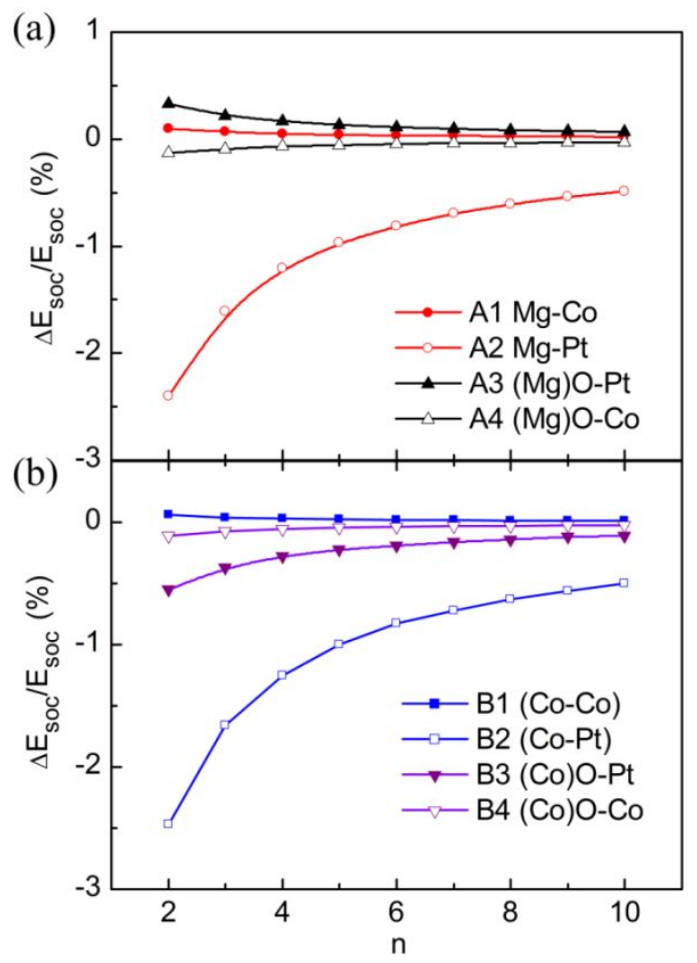

Fig. S8 The $\Delta \mathrm{E}_{\mathrm{soc}} / \mathrm{E}_{\mathrm{soc}}$ value as a function of the period number (n) for the several interfacial configurations (a) A1 - A4 and (b) B1 - B4.

(a)

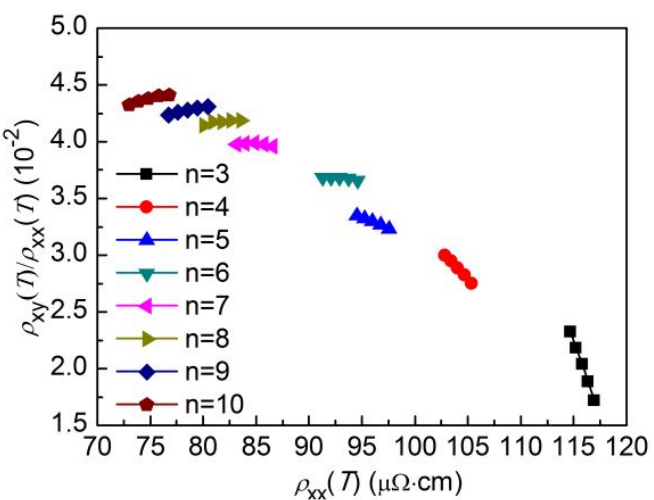

(b)

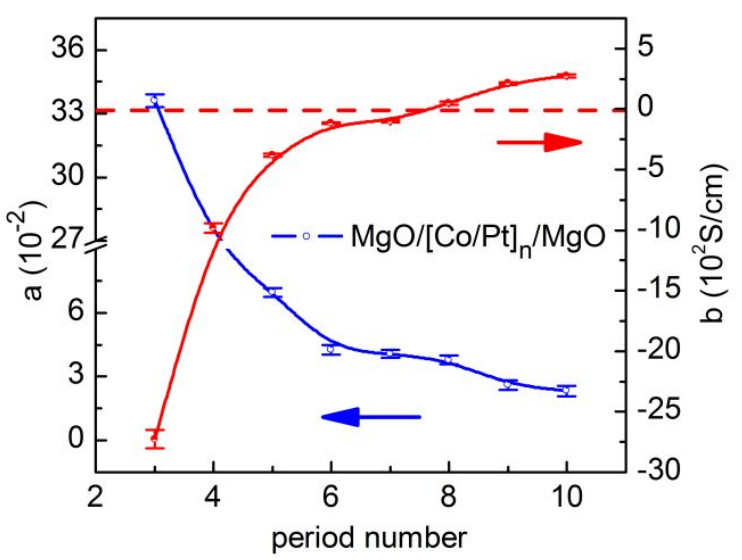

Fig. S9 (a) $\rho_{\mathrm{xy}} / \rho_{\mathrm{xx}} \sim \rho_{\mathrm{xx}}$ curves for the sample $\mathrm{MgO} /[\mathrm{Co} / \mathrm{Pt}]_{\mathrm{n}} / \mathrm{MgO}(\mathrm{n}=3 \sim 10)$. (b) The value of a and 
$\mathrm{b}$ as a function of the period number (n) for the sample $\mathrm{MgO} /[\mathrm{Co} / \mathrm{Pt}]_{\mathrm{n}} / \mathrm{MgO}(\mathrm{n}=3 \sim 10)$, respectively.

To further investigate the mechanism of AHE behavior for the $\mathrm{MgO} /[\mathrm{Co} / \mathrm{Pt}]_{\mathrm{n}}$ $/ \mathrm{MgO}$ multilayers, AHE resistance and longitudinal resistance for the multilayers were measured at various temperatures. Fig. S9 presents the relationship of $\rho_{\mathrm{xy}} / \rho_{\mathrm{xx}}$ and $\rho_{\mathrm{xx}}$ for the sample $\mathrm{MgO} /[\mathrm{Co} / \mathrm{Pt}]_{\mathrm{n}} / \mathrm{MgO}$ with different period numbers (n). For the multilayers, the $\rho_{\mathrm{xy}}$ and $\rho_{\mathrm{xx}}$ values can be obtained at a wide temperature region. The linear fit for the experimental data can be observed for the multilayers, where the intercept at $\mathrm{Y}$ axis and slope corresponds to the parameter a and b, respectively. For example, when $\mathrm{n}=3$, it (black curve) shows that a linear relationship of the $\rho_{\mathrm{xy}} / \rho_{\mathrm{xx}} \sim \rho_{\mathrm{xx}}$, where the value of $\mathrm{a}$ and $\mathrm{b}$ is $33.6 \times 10^{-2}$ and $27.3 \times 10^{-2} \mathrm{~S} / \mathrm{cm}$, respectively. The value of $\mathrm{a}$ and $\mathrm{b}$ as a function of $\mathrm{n}$ for the $[\mathrm{Co} / \mathrm{Pt}]_{\mathrm{n}}$ multilayers sandwiched by $\mathrm{MgO}$ layers were plotted in Fig. S9 (b), respectively. The value of a decreases monotonously with increasing $\mathrm{n}$. It shows a great change of a from $33.6 \times$ $10^{-2}$ to $2.3 \times 10^{-2}$. However, a nonlinear change for the value of $b$ was observed with increasing $n$. For example, the $b$ value is $-27.3 \times 10^{2} \mathrm{~S} / \mathrm{cm}$ with $\mathrm{n}=3$, but it reaches 28 $\times 10^{2} \mathrm{~S} / \mathrm{cm}$ with $\mathrm{n}=10$. Moreover, the sign of $\mathrm{b}$ has a significant change with increasing $\mathrm{n}$. It is opposite for $\mathrm{a}$ and $\mathrm{b}$ with lower $\mathrm{n}$ (below 7 ), indicating that the relationship between skew scattering (SS) and side jump scattering (SJ) should be competitive with $\mathrm{n} \leq 6$. 